\title{
COMUNIDAD ANDINA DE NACIONES (CAN), PERÚ, COLOMBIA, BOLIVIA Y ECUADOR: PROCESO DE CONVERGENCIA Y ADOPCIÓN DE LAS NIIF
}

\section{ANDEAN COMMUNITY OF NATIONS (CAN), PERU, COLOMBIA, BOLIVIA AND ECUADOR: THE PROCESS OF CONVERGENCE AND ADOPTION OF NIIF}

\author{
Cristina Pérez Rico ${ }^{1}$ \\ Universidad de Cuenca \\ cristina.perez@ucuenca.edu.ec \\ Vicente Méndez Rojas ${ }^{1}$ \\ Universidad de Cuenca \\ vicente.mendez@ucuenca.edu.ec
}

\author{
Carlos Fernández García' \\ Universidad de Cuenca \\ carlos.fernandez@ucuenca.edu.ec \\ María Alvarado Riquelme ${ }^{2}$ \\ Universidad Rey Juan Carlos (ES) \\ maria.alvarado@urjc.es
}

\author{
Paola Méndez Rojas ${ }^{1}$ \\ Universidad de Cuenca \\ paola.mendez@ucuenca.edu.ec
}

\section{Resumen}

El presente trabajo es una aproximación académica a los procesos recientes de adopción de las Normas Internacionales de Información Financiera (en adelante NIIF) de los países miembros de la Comunidad Andina (en adelante CAN) formada por Bolivia, Colombia, Ecuador y Perú, y sus similitudes $y$ diferencias, utilizando fuentes primarias $y$ secundarias, entre las que se encuentran, leyes, resoluciones de los organismos de control, documentos técnicos del IASB (International Accounting Standard Board), artículos científicos y trabajos de fin de grado de los países objeto de estudio. Se hace una revisión actualizada de los procesos de adopción de las NIIF por parte de los países de la CAN, resaltando la República del Ecuador, donde se revisaron documentos técnicos del Instituto de Investigaciones Contables del Ecuador (IICE) y de la Federación Nacional de Contadores del Ecuador (FNCE) elaborados previamente al proceso.

\section{Palabras claves}

Comunidad Andina, Normas Internacionales de Información Financiera, NIIF, adopción, estándares internacionales, contabilidad, integración.

\begin{abstract}
This document provides an approximate academic approach to the current adoption process of International Financial Reporting St andards (IFRS) of countries that form Andean Comunity of Nations (CAN) the countries included are: Bolivia, Colombia, Ecuador y Perú. This document describes the similarities and the differences of the countries that form the Andean Community. We use primary and secondary sources such laws, control institutions resolutions and work documents of IASB (International Accounting Standard Board), research articles and dissertations of the countries mentioned, are also included. This project is made with an actual current review of the adoption process of the Andean Community's countries, focusing on the Republic of Ecuador where we analyzed work documents of the Accountancy Research Institute of Ecuador (IICE) and National Federation of Accountants of Ecuador (FNCE), previously the NIIF adoption.
\end{abstract}

\section{Keywords}

Andean Comunity, International Financial Reporting Standards, IFRS, adoption, international standards, accountancy, integration.

1 Facultad de Ciencias Económicas y Administrativas de la Universidad de Cuenca (Ecuador).

2 Facultad de Ciencias Jurídicas y Sociales de la Universidad Rey Juan Carlos (España). 


\section{Introducción}

La CAN tiene su origen en el Pacto Andino o Acuerdo de Cartagena que tuvo lugar el 26 de mayo de 1965 donde se fijaron como objetivos la integración y la cooperación económica y social entre los países firmantes. Pero no fue hasta el año 1997 cuando se creó la Comunidad Andina como una actualización del Pacto Andino del año 1965 con los miembros actuales: Perú, Colombia, Bolivia y Ecuador (Comunidad Andina de Naciones, 2014). Los países integrantes de la CAN se caracterizan por una gran desigualdad en la distribución de la riqueza (Díaz-Silveira Santos, 2008), y por ello en el año 2007, en la Cumbre de Tarija, los Presidentes de la Comunidad Andina acordaron impulsar una integración plena entre la que se encuentra la económica, para la cual es indispensable disponer de un lenguaje común en materia contable.

Para la comparabilidad de la información financiera es necesario partir de un mismo marco normativo como el que nos ofrecen las NIIF. Los procesos a adopción o adaptación que se han vivido y que se están viviendo en los países de la región Andina van a contribuir a la consecución de uno de los principios de la Comunidad Andina, la integración económica regional (Morán Rosado, 2008). En el año 2007 el IFAC realizó una encuesta en la que el $89 \%$ de los encuestados consideraba que la convergencia hacia el uso de estándares contables internacionales era muy importante y clave para el desarrollo económico (Rezaee, Smith, \& Szendi, 2010).

Además la adopción de las NIIF contribuye a alcanzar uno de los principales desafíos que tiene la integración latinoamericana según J. M. Morán Rosado (2008), que es la diversificación de la inversión extranjera. La adopción de la contabilidad a estándares internacionales atrae inversión debido al uso de un mismo marco normativo y a la existencia de una información financiera mucho más transparente y profunda, lo que provoca un ahorro de costes en las empresas multinacionales y las "multipaís".
Esta prioridad ya fue expuesta por la Organización Mundial del Comercio (OMC) en el año 1995 como vía para reafirmar la internacionalización de la economía o por el Banco Interamericano de Desarrollo (BID) que ha apoyado estas iniciativas desde los años 90 a través del programa de financiación "FOMIN" (Universidad de Medellín et al., 2008).

Actualmente hay más de cien países que usan las Normas Internacionales de Información Financiera como marco contable, las cuales han sido integradas en los usos contables de cada país de diferente forma. De manera esquemática las formas de aplicar las NIIF en América Latina han sido las siguientes (Colmenárez, A., Pinto, F., Mena, R., García, 2013):

a) Adopción plena para todas las empresas pero en un momento determinado del tiempo, sin actualización posterior.

b) Adopción de las normas emitidas por el IASB en un momento determinado.

c) Adopción plena para las empresas de interés público y aplicación de las normas internacionales para el resto.

\section{d)Adopción plena para todas las empresas.}

En este artículo van a ser revisados los procesos de adopción de las NIIF en los países integrantes de la CAN a través de fuentes secundarias disponibles. 


\section{Antecedentes sobre las NIIF}

El objetivo del IASB es el de desarrollar un conjunto único de normas de información financiera legalmente exigibles y globalmente aceptadas, comprensibles y de alta calidad basadas en principios claramente articulados.

Las Normas Internacionales de Información Financiera o IFRS (International Financial Reporting Standars) están construidas a partir de principios y esa es su diferencia fundamental en relación con los anteriores sistemas de contabilidad basados en reglas.

El sistema de IFRS está compuesto por los estándares internacionales de información financiera, los estándares internacionales de contabilidad (NIC, Normas Internacionales de Contabilidad) y las interpretaciones emanadas por el Comité de Interpretaciones de las Normas Internacionales de Información Financiera (CNIIF) o del anterior Comité de Interpretaciones (SIC).

Dependiendo de quiénes los apliquen se encuentran tres grandes grupos: NIIF plenas (full IFRS), NIIF para PYMES e IPSAS (NIIF para el sector público).

Desde el año 2001 casi 114 países en el mundo han requerido o permitido el uso de las NIIF para todas o casi todas las empresas de su jurisdicción (Pacter, 2015). Entre ellos se encuentran los países de la Unión Europea, Australia, Nueva Zelanda, Rusia, Hong Kong, Costa Rica, Uruguay, Perú, China, India, Ecuador, etc. Por su parte en el año 2002 los Estados Unidos a través del FASB (Financial Accounting Standards Board) firmó un convenio con el IASB para la convergencia de los USGAAP ( United States Generally Accepted Accounting Principles) con las NIIF.

Por último, cabe señalar la importancia del Grupo Latinoamericano de Emisores de Información Financiera (GLENIF), del que es miembro Bolivia, Colombia, Ecuador y Perú. Este grupo es el representante de los países latinoamericanos frente al International Accounting Standards Board (IASB) en temas contables y este promueve la adopción y/o convergencia de las NIIF, apoyando a gobiernos, organismo reguladores y otras organizaciones que contribuyan al mejoramiento de la calidad financiera de los estados financieros de la región contribuyendo a la difusión de las normas emitidas por el IASB (GLENIF, s. f.). Algunos beneficios de adoptar las NIIF son (Mantilla, 2012):

- Lograr comparabilidad internacional de la información financiera.

- Mejorar la comprensibilidad de los estados financieros tanto a nivel nacional como internacional.

- Mayor aceptación y confianza de los estándares por los proveedores de capital en los diferentes países donde se apliquen.

- Mejorar la competencia de los negocios a nivel internacional por la reducción de los costos de cumplimiento.

- Reducir los costos administrativos al presentar estados financieros más cercanos a la realidad.

Pero este proceso también ha tenido problemas en todas las jurisdicciones donde se ha implementado, incluidos los países que conforman la CAN. Estos problemas tienen que ver con la armonización de las NIIF con los estándares contables nacionales y las leyes tributarias (Bozkurt, İslamoğlu, \& Öz, 2013). 
Experiencias de adopción en los países miembros de la CAN

\section{Proceso de adopción de las NIIF en Colombia}

En Colombia el proceso de adopción de las NIIF está siendo largo y aún no ha concluido, esto ha traído como consecuencia el retraso en la incorporación del país a diversos convenios o tratados internacionales que imponen como condición el uso de las NIIF.

En el año 1993 se reglamentó la contabilidad y en el texto resultante se adoptaron las Normas Internacionales de Contabilidad (NIC) vigentes en aquel momento para facilitar la comprensión y mejorar los estándares de calidad de la información financiera a nivel de país. De esta reglamentación surgió el Plan Único de Cuentas o PUC (Luna, 2011) incluido en el Decreto 2650 del año 1993. Éste sigue vigente en la actualidad y consiste en un catálogo de cuentas de obligado uso, su descripción y funcionamiento.

No obstante las normas emitidas por el IASB continúan actualizándose, mientras que en Colombia quedaron estáticas y al poco tiempo obsoletas, sufriendo por ello presiones de la Organización Mundial del Comercio a la cual pertenece Colombia.

En 1999 el Congreso de Colombia emitió la Ley 550 de 1999 donde en su artículo 63 se comprometía a revisar las normas contables y de información financiera con el fin de ajustarlas a los parámetros internacionales (Ferrer De La Hoz, 2013). No fue hasta finales del año 2003 cuando el gobierno, a través de Comité Técnico, preparó un Proyecto de Intervención Económica, que entre sus objetivos constaban el adoptar las NIIF y a partir del año 2006 las Normas Internacionales de Auditoría (NIA). Todo esto generó un intenso debate sobre si era mejor adoptar las NIIF o no adoptarlas y finalmente el Gobierno decidió no proseguir con el proyecto en aquel momento.
El 27 de junio del 2007 entró en vigor la Ley 1116 de 2006 que otorga al Gobierno la facultad de proponer al Congreso las modificaciones necesarias para la aplicación de las NIIF en Colombia en cualquier momento y sin fecha de caducidad.

Fue después de esto que se realizó el Proyecto de Ley 165 de 2007 donde se proponía las NIIF como marco para la presentación de informes contables. Este proyecto sufrió multitud de modificaciones hasta que el 13 de Julio de 2009 fue sancionado por el presidente de la República, que dio lugar a la Ley 1314 de 2009 (KPMG, 2015; Luna, 2011; Pacter, 2015).

En esta ley se estableció por el único organismo autorizado para redactar normas de convergencia que es el Consejo Técnico de Contaduría Pública que depende orgánicamente del Ministerio de Comercio, Industria y Turismo (Ferrer De La Hoz, 2013; IFRS, 2013a). Además presentó el cronograma para la adopción en tres grupos (IFRS, 2013a; Pacter, 2015):

- Grupo 1 compuesto por todas las empresas obligadas a utilizar las NIIF completas, que son las que emiten valores y las entidades de interés público principalmente. Además por el decreto 2784 también se incluyeron dentro de este grupo las empresas "subordinadas o matrices de empresas nacionales que estén obligadas a aplicar las NIIF ya sean plenas, subordinadas, matrices, asociadas o de negocios conjuntos de entidades extranjeras que apliquen las NIIF plenas y también aquellas que sus importaciones representen más del $50 \%$ de sus compras y ventas" (Ferrer De La Hoz, 2013). Las empresas de este grupo tienen la obligación de utilizar las NIIF a partir del año 2015.

- Grupo 2 compuesto por todas las empresas que utilizarán las NIIF para PYMES que son las empresas de tamaño grande y mediano que no están en el grupo 1. Se comenzará a usar las NIIF a partir del año 2016.

- $\quad$ Grupo 3 formado por las empresas clasificadas como pequeñas y microempresas 
bajo la clasificación legal colombiana de empresas. Estas empresas utilizarán las Normas de Información Financiera para Microempresas.

En la actualidad el Comité de Sistema Documental Contable se está planteando el desafío de implementar el lenguaje XBRL asesorado por la Fundación XBRL España (Ferrer De La Hoz, 2013) que consiste en implementar un sistema estándar de presentación de la información financiera y empresarial en formato electrónico.

\section{Proceso de adopción de las NIIF en Bolivia}

El órgano encargado de elaborar y emitir las normas contables en Bolivia es el Consejo Técnico Nacional de Auditoría y Contabilidad (CTNAC) que depende del Colegio de Auditores de Bolivia (CAUB) (IFRS, 2013b; Vásquez, 2013).

En Bolivia la primera muestra de interés por la convergencia hacia las NIIF fue en el año 1994 cuando el CTNAC emitió la resolución 02/94 donde estableció la adopción de las NIC para todos aquellos hechos económicos no tratados por las normas locales, y de esa forma cubrir aquellos aspectos donde no existía una normatividad contable. Esta resolución fue ratificada en el año 2003 por la resolución 03-03 del mismo organismo y también en el año 2007 en la NC3 de estados financieros a moneda constante (Quispe Caballero, 2011).

En el año 2007 el Banco Interamericano de Desarrollo (BID) y el Colegio de Auditores de Bolivia (CAUB) suscribieron un convenio de cooperación técnica, el cual fue financiado por el BID que entregó una subvención no reembolsable para el proyecto que tenía entre sus objetivos la creación de normas bolivianas armonizadas con las normas internacionales. Como uno de los resultados de este convenio, en el 2010 se obtuvieron treinta y siete Normas de Información Financiera y un Marco de Conceptos para la Preparación de Estados Financieros pero aún no han entrado en vigor (Quispe Caballero, 2011). Pero ya un año antes, en el 2009, se aprobaron catorce normas de contabilidad financiera que siguen en vigor tras la emisión de las Resoluciones del Consejo Técnico Nacional de Auditoría y Contabilidad 001/2012 y 002/2012.

La NIIF nada más traducidas al español, también fueron adoptadas y siguen vigentes para todo lo no contemplado por las catorces normas anteriores (Bolivia-CAUB, 2013).

Actualmente, se ha aprobado por el CTNAC un cronograma para la implementación de las NIIF, que comenzará el año 2015 y terminará en el 2017. No obstante hasta que no sea aprobado por la Autoridad de Fiscalización y Control Social de Empresas (AEMP) la aplicación no es firme (Pacter, 2015).

\section{Proceso de adopción de las NIIF en Ecuador}

En el año 1996 la FNCE (Federación Nacional de Contadores del Ecuador) como miembro del IFAC (International Federation of Accountants) con resolución FNCE 09.01.96 del 28 de septiembre de 1996 adoptó las NIC (Normas Internacionales de Contabilidad) como base para la emisión de las NEC (Normas Ecuatorianas de Contabilidad (IICE y FNCE, 2009; Vásquez, 2013). Las NIC son las primeras normas emitidas por el organismo internacional IASB que están en constante revisión y evolución para adaptarse a las necesidades y circunstancias de los usuarios de la información financiera.

El problema que tuvieron las NEC es que no se actualizaron a la misma velocidad que la normativa internacional, y llegó un momento que estaban obsoletas. Esto se convirtió en un problema para la comparabilidad de los estados financieros de las empresas ecuatorianas con el resto de empresas de otros países, fracasando en el objetivo con el que nacieron las NEC que era el de seguir la tendencia mundial hacia la convergencia internacional y facilitar la comprensión de los estados financieros ecuatorianos para los 
inversores extranjeros consiguiendo una mayor comparabilidad y transparencia de la contabilidad ecuatoriana.

La Superintendencia de Compañías es el organismo responsable de la vigilancia y control de las compañías constituidas en el Ecuador, dentro de las cuales se encuentran las compañías en nombre colectivo, las compañías en comandita simple y dividida por acciones, las compañías de responsabilidad limitada, las compañías anónimas y las compañías de economía mixta (Congreso, 2014). Este organismo, el 26 de agosto del año 2006 sustituye las NIC (Normas Internacionales de Contabilidad) por las NEC y las NIA (Normas Internacionales de Auditoría) por las NEA (Normas Ecuatorianas de Auditoría) (Superintendencia de Compañías, 2006a, 2006b). La razón fundamental para este cambio es la aplicación de criterios uniformes en la presentación de la información financiera y la correspondiente evaluación a través de la auditoría, facilitando la integración comercial internacional.

El 11 de octubre del año 2011 la Superintendencia de Compañías estableció el Reglamento para la aplicación de las NIIF completas y para PYMES (Superintendencia de Compañías, 2011), que continua vigente en la actualidad.

Para efectos del registro y preparación de estados financieros, la Superintendencia de Compañías califica como PYMES a las personas jurídicas que cumplen todas las siguientes condiciones:

a) Monto de Activos inferiores a cuatro millones de dólares.

b) Empresas que registren un Valor Bruto de Ventas Anuales de hasta cinco millones de dólares.

c) Empresas que tengan menos de 200 trabajadores (personal ocupado). Para este cálculo se tomará el promedio anual ponderado.
Se considerará como base los estados financieros del ejercicio económico el año anterior al periodo de transición, es decir, que si el año de transición fuera el 2010, el año considerado como base sería el 2009.

Si a partir del año 2011 una empresa que adoptaba las NIIF completas cumple con los requisitos necesarios para ser denominada PYME podrá optar por implementar las NIIF para PYMES. El periodo de transición para las compañías que cambiaron su situación fue el año del cambio.

A nivel de las empresas del sector público, existen diferentes disposiciones relacionadas con la aplicación de las NIIF. La Superintendencia de Compañías, establece que las empresas bajo su control están obligadas a la aplicación, por lo tanto las empresas de economía mixta y las empresas que se mantienen con otra denominación, estarían dentro de este marco legal (Congreso, 2014). En el caso de las empresas públicas, constituidas al amparo de la Ley Orgánica de Empresas Públicas, se establece que la contabilidad estará basada en los principios de general aceptación y en las normas internacionales de contabilidad (Pleno De La Comision Legislativa Y De Fiscalizacion, 2009), disposición que concuerda con lo establecido por el Ministerio de Finanzas, para las empresas públicas que requieran la devolución del IVA, quienes deben presentar contabilidad basada en los principios de contabilidad de general aceptación y NIIF (Ministerio Finanzas, 2012).

Cabe señalar la publicación del Memorando No. SC.Q.ICI.CPAIFRS.11.009 el 21 de enero del 2011, que consiste en un instructivo de estados financieros bajo NIIF, donde se explica cómo deberán elaborar las empresas los formularios obligatorios de estado de situación financiera, estado de resultado integral, estado de flujos de efectivo por el método directo y estado de cambios en el patrimonio. Además se incluye una descripción de cada una de las partidas que integran cada uno de los estados financieros (Superintendecia de Compañías, 2011). 


\section{Proceso de adopción de las NIIF en Perú}

El proceso de adopción de las NIIF comenzó en Perú en el año 1998, cuando el Consejo Normativo de Contabilidad en su Resolución $\mathrm{N}^{\circ}$ 13 estableció que los principios de contabilidad generalmente aceptados del país comprendían a las Normas Internacionales de Contabilidad y supletoriamente los principios de contabilidad aplicados en los Estados Unidos de América (USGAAP) (Morales Cano, 2010). No obstante, esta resolución no sirvió para que las empresas adoptaran las NIIF completamente, por lo que el 14 de octubre del 2010, la Comisión Nacional Supervisora de Empresas y Valores (CONASEV), actualmente Superintendencia de Mercado de Valores (SMV) mediante su Resolución No 102, dispuso que las empresas que estaban bajo su supervisión presentaran sus estados financieros bajo NIIF (Vásquez, 2013), según un cronograma en el que establecía que en el año 2011 tenían la obligación de presentar las cuentas bajo este marco normativo internacional, las sociedades emisoras de valores inscritas en el Registro Público del Mercado de Valores (RPMV), las empresas clasificadoras de riesgo y las demás empresas jurídicas supervisadas por la SMV, y en el año 2012, las bolsas de valores, las instituciones de compensación y liquidación de valores, los agentes de intermediación, las sociedades administradoras de fondos mutuos de inversiones en valores, los fondos de inversión y las sociedades titulizadoras.

Asi mismo el 9 de junio del 2011, la Ley 29720 estableció que las sociedades distintas a las que se encuentran bajo supervisión de la SMV cuyos ingresos anuales o sus activos totales sean iguales o excedan a 3.000 Unidades Impositivas Tributarias (UIT), deben presentar a esa entidad sus estados financieros conforme a las NIIF, auditados y con acceso al público. Para precisar esta ley y establecer un cronograma de aplicación, el 27 de abril del 2012 la SMV emitió la Resolución N ${ }^{a} 11$, donde estableció que las empresas que superen las 30.000 UIT de ingresos anuales o activos totales al cierre del ejercicio del año 2012 (40 millones de dólares al cierre del 2011) deberán aplicar las NIIF en el periodo del año 2013. El resto de empresas con un máximo de cuatro millones de dólares de activos deben preparar sus estados contables de acuerdo con las NIIF entre los años 2014 a 2017 en función de sus ingresos y activos (KPMG, 2015).

\section{Análisis comparativo de los procesos.}

En todos los países de la CAN se comenzaron los procesos de acercamiento a la adopción de normas internacionales emitidas por el IASB en la década de los 90 . El primer país en hacerlo fue Colombia en el año 1993, seguido de Bolivia en 1994, Ecuador en 1996 y Perú en 1998.

Colombia es el único país que ha regulado el proceso mediante ley, por lo que su proceso goza de una mayor institucionalización otorgado por la jerarquía legislativa colombiana, donde las leyes solo están por debajo de la Constitución.

En un primer momento en Perú se emitió una resolución para regular el proceso para las empresas sujetas a la Superintendencia de Mercado de Valores en el año 2010. Después en el año 2011 se amplió mediante ley, la población de empresas obligadas a adoptar las NIIF a todas aquellas que tuviesen más de 3.000 Unidades Impositivas Tributarias de ingresos o activos, dando al proceso una mayor amplitud y una mayor fuerza legislativa.

En Ecuador el proceso está regulado por resoluciones emitidas por la Superintendencia de Compañías que es el organismo de control de las empresas dentro de la República.

Como último caso estaría el de Bolivia donde aún no se ha designado el organismo encargado del proceso para la emisión de normativas para la adopción de las NIIF, por lo que existe un vacío legal. En las resoluciones del CTNAC № 001/2012 y $\mathrm{N}^{\circ} 002 / 2012$, de manera residual se establece que en ausencia de legislación local en alguna materia se puede utilizar las Normas Internacionales de Información Financiera. En Bolivia es donde las 
NIIF están menos institucionalizadas y tienen menos fuerza legislativa. En Ecuador el proceso de adopción ya ha concluido para las empresas establecidas. En Colombia y en Perú aún no ha concluido el proceso de adopción y en Bolivia solo se han dado los primeros pasos hacia el comienzo del proceso.

\begin{tabular}{|c|c|c|c|c|}
\hline & Colombia & Bolivia & Ecuador & Perú \\
\hline $\begin{array}{c}\text { Forma de aplicar las NIIF } \\
\text { en la actualidad }\end{array}$ & $\begin{array}{l}\text { En el año } 2015 \text { entra en } \\
\text { vigor el uso de las NIIF } \\
\text { para las empresas del } \\
\text { primer grupo del } \\
\text { cronograma que deben } \\
\text { aplicar NIIF plenas. }\end{array}$ & $\begin{array}{c}\text { Resoluciones del CTNAC N } \\
001 / 2012 \text { y } \mathrm{N}^{\circ} 002 / 2012 \\
\text { establecen la adopción de las } \\
\text { Normas Internacionales de } \\
\text { Información Financiera en } \\
\text { ausencia de legislación local. }\end{array}$ & $\begin{array}{l}\text { Adopción de las NIIF desde } \\
\text { el momento en que se } \\
\text { produce su traducción al } \\
\text { español. }\end{array}$ & $\begin{array}{l}\text { Adopción de las NIIF para } \\
\text { todas las empresas marcadas } \\
\text { por la ley. }\end{array}$ \\
\hline $\begin{array}{c}\text { Año de creación de primer } \\
\text { acercamiento a normativa } \\
\text { internacional }\end{array}$ & $\begin{array}{c}\text { En el año } 1993 \text { cuando se } \\
\text { reglamentó la contabilidad } \\
\text { de acuerdo con las NIC. }\end{array}$ & $\begin{array}{c}\text { En el año } 1994 \text { cuando se } \\
\text { emitió la resolución } 02 / 94 \text { que } \\
\text { estableció la adopción de las } \\
\text { NIC para todos los aspectos } \\
\text { no recogidos por normas } \\
\text { locales. } \\
\end{array}$ & $\begin{array}{c}\text { En el año1996, FNCE emitió } \\
\text { resolución 09.01.96 donde se } \\
\text { adoptaron las NIC como base } \\
\text { para las NEC. }\end{array}$ & $\begin{array}{l}\text { En el año } 1998 \text { cuando se } \\
\text { aprobó la Resolución } 13 \text { que } \\
\text { estableció las NIC como } \\
\text { principios de contabilidad. }\end{array}$ \\
\hline $\begin{array}{l}\text { Ley que aprobó la } \\
\text { aplicación de las NIIF }\end{array}$ & Ley 1314 del año 2009 & No hay ley & $\begin{array}{l}\text { Resolución No. } \\
\text { SC.ICI.CPAIFRS.G.11 de } 11 \\
\text { de octubre del año } 2011 .\end{array}$ & $\begin{array}{c}\text { Resolución } \mathrm{N}^{\circ} 102 \text { del año } \\
2010 \text { para las empresas bajo la } \\
\text { supervisión SMV, y Ley } \\
29720 \text { del año } 2011 \text { para todas } \\
\text { las empresas cuyos ingresos o } \\
\text { activos totales sean o superen } \\
\text { 3.000 Unidades Impositivas } \\
\text { Tributarias. }\end{array}$ \\
\hline Estado de la adopción & En proceso de adopción. & $\begin{array}{c}\text { Aún no se ha comenzado } \\
\text { proceso. }\end{array}$ & $\begin{array}{l}\text { Adopción plena de todas las } \\
\text { empresas sujetas a la } \\
\text { Superintendencia de } \\
\text { Compañías. }\end{array}$ & $\begin{array}{l}\text { Proceso concluido para las } \\
\text { empresas que están bajo el } \\
\text { control de la SMV y que } \\
\text { igualan o superan las } 3.000 \\
\text { Unidades Impositivas o } \\
\text { Tributarias en sus activos o } \\
\text { ingresos. } \\
\end{array}$ \\
\hline
\end{tabular}

\section{Conclusiones y futuras líneas de investigación}

Los procesos de adopción de las NIIF en los países de la CAN han sido complicados por la falta de apoyo de las instituciones en materia de formación, que han tenido como consecuencia la carencia de guías técnico-contables, de guías en la primera adopción de NIIF y de personal cualificado (Vásquez, 2013). Las transformaciones de las normas contables han sido muy rápidas y no han contado con el apoyo de todos los agentes implicados. Aunque el marco legislativo apoya el uso de la normativa internacional en la práctica todavía falta mucho para conseguir la convergencia definitiva ya que sólo se han dado los primeros pasos. En algunos países de la CAN, como es el caso de Colombia o Perú, la aplicación de estos procesos se ha hecho mediante leyes, lo que proporciona una mayor fuerza coercitiva, lo que ha significado una institucionalización de las Normas Internacionales de Información Financiera.

Además, en el caso de Colombia, la existencia del Plan Único de Cuentas ayuda a la uniformidad de los registros contables facilitando su comprensión y la práctica contable. Debido a los buenos resultados obtenidos en Colombia con la implantación del PUC, los organismos encargados 


\section{Cristina Pérez Rico / Carlos Fernández García Vicente Méndez Rojas / Paola Méndez Rojas / María Alvarado Riquelme.}

de la normalización de los procesos contables en cada uno de los países de la CAN deberían de emitir un Plan Único de Cuentas propio adaptado a sus necesidades. En el caso concreto de Ecuador, sería un buen punto de partida el Memorando No. SC.Q.ICI.CPAIFRS.11.009, que consta de una descripción de cada una de las partidas de los estados financieros.

La complejidad de las Normas Internacionales de Información Financiera impone una profesionalización mucho más intensa de los contadores y auditores. La permanente modificación de las normas obliga a estos profesionales a una continua actualización de conocimientos que en la práctica no siempre se realiza por los costes asociados.

En este momento, se les impone como obligación a las universidades asumir el rol de cubrir las carencias formativas, cambiando mallas y sílabos, para conseguir transformar el uso de la contabilidad en un instrumento financiero que ayude a la toma de decisiones de los usuarios de la información financiera, separando la contabilidad de la fiscalidad, tal y como establece el IASB (Ferrer De La Hoz, 2013).

Además los colegios profesionales, apoyados por las universidades, deben de contribuir al apoyo de la actualización contable y educación continua de los profesionales de la zona andina para que la plena convergencia sea una realidad.

Por último es necesario señalar la necesidad de que todos los países de la zona andina pongan en su agenda el conocimiento y uso del XBRL (Extensible Bussines Reporting Lenguage) que consiste en un lenguaje de programación que pretende unificar los estándares aplicables a la presentación de información financiera electrónica.

\section{Bibliografía}

Colegio de Auditores de Bolivia (2013). Compendio de Normas de Contabilidad y Auditoría.

Bozkurt, O., İslamoglu, M., \& Öz, Y. (2013). Perceptions of professionals interested in accounting and auditing about acceptance and adaptation of global financial reporting standards. Percepciones de profesionales interesados en contabilidad y auditoría acerca de la aceptación y adaptación de normas internacionales de información financiera., 18(34), 16-23. doi:http://dx.doi.org/10.1016/S2077-1886(13)70019-2

Colmenárez, A., Pinto, F., Mena, R., García, J. (2013). Problemas y experiencias en la adopción de normas internacionales de información financiera por primera vez en países de América. En XXX Conferencia Interamericana de Contabilidad.

Comunidad Andina de Naciones. (2014). Comunidad Andina. Recuperado 30 de septiembre de 2014, a partir de http:// www.comunidadandina.org/Seccion.aspx?id=195\&tipo=QU\&title=resena-historica

Congreso. Ley de compañías (2014). Ecuador.

Díaz-Silveira Santos, C. (2008). La cohesión social: el reto actual de las relaciones entre la Unión Europea y los procesos de integración latinoamericanos. Revista Electrónica Iberoamericana, 2, 99-110.

Ferrer De La Hoz, A. M. (2013). Análisis del proceso de convergencia a Normas Internacionales de Contabilidad e Información Financiera desde los factores intrínsecos al sistema contable en Colombia. Cuadernos de Contabilidad, 14(36), 971-1007. Recuperado a partir de http://repository.unimilitar.edu.co:8080/bitstream/10654/10227/1/ MartinezSerpaFabianAlfonso2013.pdf 
GLENIF. (s. f.). Nuestro objetivo. Recuperado 13 de junio de 2015, a partir de http://glenif.org/es/index.php?option=com_ content\&view $=$ article\&id=53\&/temid $=62$

IFRS. (2013a). IFRS APPLICATION AROUND THE WORLD JURISDICTIONAL PROFILE : Colombia. doi:http://www.ifrs. org/Use-around-the-world/Documents

IFRS. (2013b). IFRS application around the world jurisdictional Profile: Bolivia. Recuperado a partir de http://www.ifrs. org/Use-around-the-world/Documents/Jurisdiction-profiles/Bolivia-IFRS-Profile.pdf

IICE y FNCE. (2009). Plan de adopción y socialización en el Ecuador de las Normas Internacionales de Información Financiera y de las Normas Internacionales de Auditoria y Aseguramiento. Guayaquil.

KPMG. (2015). Aplicación de las Normas Internacionales de Información Financiera en Latinomérica.

Luna, L. J. (2011). Colombia: hacia la adopción y aplicación de las NIIF y su importancia. Revista Virtual de Estudiantes de Contaduría Pública, (8), 26-43.

Mantilla, S. A. (2012). Estándares/Normas Internacionales de Información Financiera IFRS (NIIF) (Tercera ed). Bogotá: Ecoe Ediciones.

Ministerio Finanzas. Acuerdo ministerial 037 (2012). Ecuador.

Morales Cano, A. M. (2010). Algunas experiencias exitosas en Latinoamérica relacionadas con la estandarización contable. Revista Facultad de Ciencias Económicas: Investigación y Reflexión, XVIII(2), 207-226.

Morán Rosado, M. J. (Universidad de E. (2008). Los retos básicos del proceso de integración en lberoamérica. Revista Electrónica Iberoamericana, 2(1), 39-48.

Pacter, P. (2015). IFRS Foundation $₫$ IFRS $₫$ as global standards : a pocket guide.

Pleno De La Comision Legislativa Y De Fiscalizacion. (2009). Ley Organica De Empresas Publicas, 92. Ecuador.

Quispe Caballero, Á. M. (Universidad M. de "San A. (2011). Aplicación de la norma internacional de información financiera para pequeñas y medianas empresas en Bolivia. Universidad Mayor de "San Andrés».

Rezaee, Z., Smith, L. M., \& Szendi, J. Z. (2010). Convergence in accounting standards: Insights from academicians and practitioners. Advances in Accounting, 26(1), 142-154. doi:10.1016/j.adiac.2010.01.001

Superintendencia de Compañías. (2011). Memorando No. SC.Q.ICI.CPAIFRS.11.009. Ecuador.

Superintendencia de Compañías. No. 06.Q.ICI.003 (2006). Ecuador.

Superintendencia de Compañías. No. 06.Q.ICI.004 (2006). Ecuador.

Superintendencia de Compañías. No. SC.ICI.CPAIFRS.G.11 (2011). Ecuador.

Universidad de Medellín et al. (2008). Banco de experiencias existosas en la aplicación de las Normas Internacionales de Información Financiera (NIIF/NIC): en los países de Mercosur, Comunidad Andina, México y España.

Vásquez, N. et al. (2013). Estado actual de Latinoamérica en relación a su proceso de adopción de las NIIF. Contabilidad y Auditoría Investigaciones en Teoría Contable de la Sección de Investigaciones Contables, (39), 55-103. 